\title{
High precision and tunable multi-wavelength fiber source based on cascaded four-wave mixing enhanced by Raman
}

\author{
Ana Carrasco-Sanz ${ }^{* a}$, Sonia Martín-López ${ }^{\mathrm{a}}$, Miguel González-Herráez ${ }^{\mathrm{b}}$, Pedro Corredera ${ }^{\mathrm{a}}$, Laura \\ Abrardi $^{\mathrm{a}}$, María Luisa Hernanz ${ }^{\mathrm{a}}$, Félix Rodríguez ${ }^{\mathrm{a}}$ \\ ${ }^{a}$ Dept. de Metrología, Instituto de Física Aplicada, CSIC,. Serrano 144, 28006, Madrid, Spain; \\ ${ }^{\mathrm{b}}$ Dept. de Electrónica, Escuela Politécnica Superior, Universidad de Alcalá. 28871, Alcalá de \\ Henares. Spain
}

\begin{abstract}
We present a multi-wavelength fiber source based on cascaded of four-wave mixing in two semiconductor optical amplifiers followed by further four-wave mixing in an optical fiber enhanced by Raman amplification. The multiwavelength source is generated by two initial frequencies detuned $200 \mathrm{GHz}$ and referenced in the absorption lines of the acetylene ${ }^{12} \mathrm{C}_{2} \mathrm{H}_{2}$, which sweep in frequency keeping the detuning of the lasers constant. With this configuration, we have achieved a high resolution source with a spectrum of 36 channels centered with adjustable peaks separation. The source can be employed to interrogate a fiber Bragg grating sensors network and in gas spectroscopy applications.
\end{abstract}

Keywords: multi-wavelength fiber laser, standard frequencies, four-wave mixing, semiconductor optical amplifier, Raman amplification

\section{INTRODUCTION}

Multi-wavelength laser sources present a great interest in optical sensing, optical spectroscopy, microwave signal processing, and high capacity WDM optical fiber communications. Depending on the application, the requirements for multi-wavelength sources include a large number of channels over a broad bandwidth, high output power uniformity distributed over the channels, precise position of the ITU frequency grid, high stability of their wavelength emissions or high tunability. Multi-wavelength sources are usually designed using several gain mechanisms such as erbium-doped fiber, semiconductor optical amplifier and stimulated Raman scattering, and channel spacing mechanics as diffraction gratings, Bragg gratings, Lyot filters, Sagnac filters, intracavity etalons or Fabry-Perot micro-etalons [1-7].

An interesting application of the multi-wavelength fiber source is in optical fiber sensors. Nowadays, optical fiber sensors are employed in many applications including strain measurements in civil infrastructure, oil pipeline monitoring, medical applications or chemical sensing. In many of these applications a set of Bragg gratings that forms a network much be interrogated. In many of these applications high resolution, high precision and tunability is required [8].

In this paper, we present a multi-wavelength source for interrogating a fiber Bragg grating (FBG) network using two frequencies obtained by two tunable lasers, separated $200 \mathrm{GHz}$. Both lasers sweep over the wavelength range of visible absorptions in an acetylene cell, so that there is a continuous absolute wavelength monitoring of the whole source. In order to obtain the multi-wavelength laser source the two lasers are combined to produce degenerate four-wave mixing (FWM) in a cascade of two semiconductor optical amplifiers (SOAs) and enhanced by further FWM and Raman amplification in a dispersion-shifted optical fiber. The input lasers are centered close to the zero dispersion wavelength of the optical fiber used in the experiment in other to increase the FWM efficiency, leading to the generation of up to 36 referenced lines.

\section{EXPERIMENTAL SETUP AND RESULTS}

Fig 1 shows the experimental setup used to generate the proposed multi-wavelength fiber laser. It consists of two tunable lasers, two cascade SOAs with a saturation power of 13 and $15 \mathrm{dBm}$ respectively, two isolators, two Raman fiber laser at

\footnotetext{
* carrasco@ifa.cetef.csic.es; phone +34 915618806 ext.326; fax +34 915642122
} 
wavelength $1455 \mathrm{~nm}$ and $1427 \mathrm{~nm}$ respectively, a $5 \mathrm{Km}$ dispersion-shifted fiber, an absorption acetylene cell (traceable to NIST [9]) and two optical detectors.

The system works as follows: the output of both lasers are divided in two branches. One is used to reference the laser line to the selected frequency by means of the acetylene cell, and the others are coupled for the multi-wavelength source generation. Initially, TL1 emits at the frequency of the absorption line P23, $\mathrm{f}_{1}=194.743 \mathrm{THz}(\lambda=1539.46 \mathrm{~nm})$ with output power $P_{1}=5 \mathrm{~mW}$. TL2 signal is detuned $200 \mathrm{GHz}$ from TL1, so that $\mathrm{f}_{2}=194.943 \mathrm{THz}(\lambda=1537.85 \mathrm{~nm})$ with $\mathrm{P}_{2}=$ $6 \mathrm{~mW}$. This frequency spacing can be changed depending on the requirements of the FBG network system to be interrogated. TL1 and TL2 sweep simultaneously keeping the constant detuning, and the sweep is stopped when $f_{1}$ arrives at line P27 of the acetylene absorption spectrum $(\mathrm{f}=194.386 \mathrm{THz}, \lambda=1542.25 \mathrm{~nm})$. Hence there is absolute references for TL1 in the beginning and end of the sweep, and for TL2 these appear along the sweep. Since the spectrum of acetylene is well-known, the position of these references is clearly determined along the sweep and is used as a feedback to correct any errors.

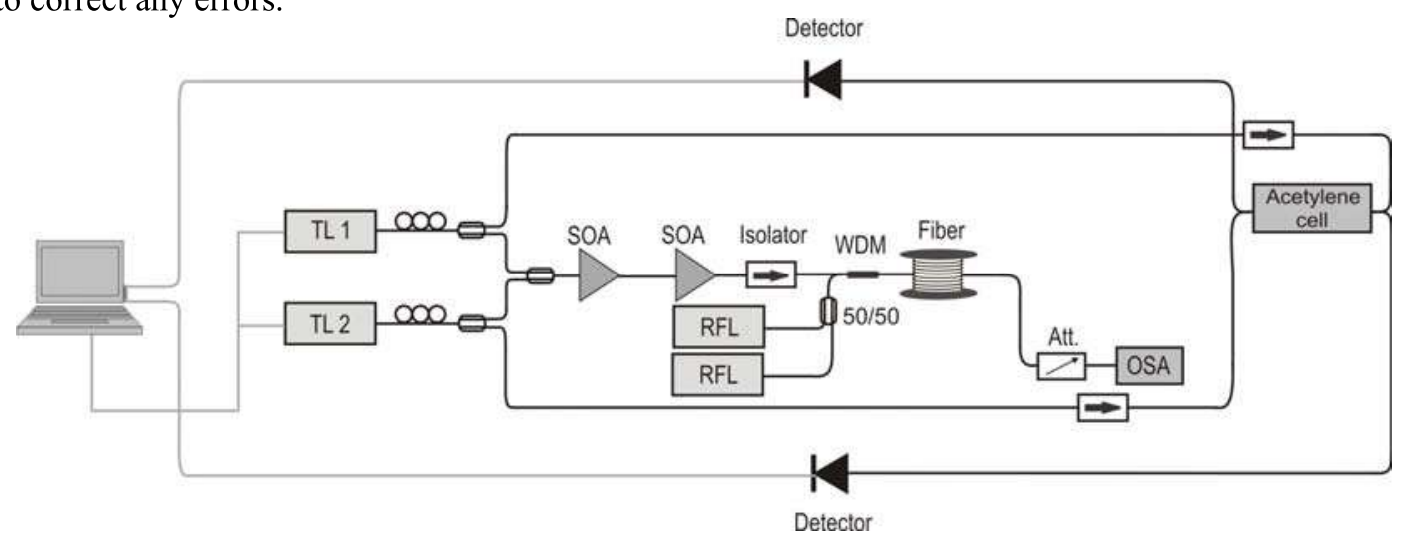

Fig 1.- Experimental setup

The signals emitted from the acetylene cell are detected by two detectors and controlled by a computer. When TL1 or TL2 emissions pass through an absorption line, the computer uses the signal detected by the detectors to correct possible frequency drifts by acting on the sweep signal of $f_{1}$ and $f_{2}$. By knowing the absorption spectrum of the acetylene [9] and assuming that the sweep is linear, the frequencies emitted from TL1 and TL2 are known in the whole process.

The signals from TL1 and TL2 are polarization-controlled to maximize the FWM efficiency and are combined at the input of the first SOA. We use two SOAs (Kamelian OPA-20-N-C-FA and Covega BOA-1004) that have longer active region than conventional ones used for amplification, and hence exhibit a larger nonlinear behavior. The two SOAs are cascaded in order to increase the output power and so as to enhance the FWM generation. Fig 2 shows the spectra obtained at the output of the first and second SOA, showing a consistent increase in the number of FWM products generated using two SOAs (nine frequencies) instead of only one SOA (six frequencies).

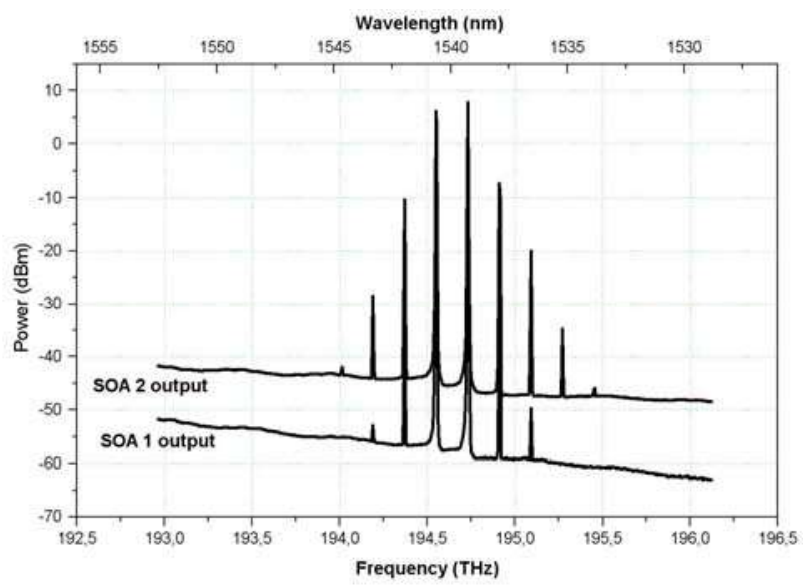

Fig 2.- FWM generated in a high non-linear SOA and enhanced in a high output saturation power SOA 
The output of the second SOA is introduced in a $5 \mathrm{~km}$ long dispersion-shifted fiber with a zero-dispersion wavelength at $1540.9 \mathrm{~nm}$ and a dispersion slope of $0.056 \mathrm{ps} \bullet \mathrm{nm}^{-2} \cdot \mathrm{km}^{-1}$. The TL1 and TL2 laser frequencies have been selected close to the zero dispersion of the fiber in order to improve the FWM generation in the fiber, and, due to its reduced effective area, the fiber also presents a relatively high Raman gain $\left(3.3 \mathrm{~dB} \cdot \mathrm{W}^{-1} \cdot \mathrm{km}^{-1}\right)$. The Raman gain is obtained in our experiment with a Raman laser centered a $1455 \mathrm{~nm}$. The benefits of introducing Raman gain in this experiment are double: first, since the input waves are amplified traveling in the fiber, the efficiency of the FWM process is largely enhanced; second, the phase matching conditions of FWM are less restrictive, as it was shown in [10]. As a consequence of this, the intensity of the generated FWM products remains basically constant over the full sweep range, which may be enough to scan the spectrum of a whole fiber Bragg grating

Fig 3 shows the output spectra obtained at the output of the fiber for different Raman pump powers. The number of visible lines increased with the Raman pump power. The optimum results are obtained for a pump power of $1 \mathrm{~W}$, case in which the optical spectra of the multiwavelength fiber laser consists of 28 equally spaced frequencies covering a range from $192 \mathrm{THz}$ to $197 \mathrm{THz}$ centered in the P23 acetylene absorption line and with a spacing of $200 \mathrm{GHz}$. As we were increasing the pump power, we could check that the number of frequencies generated did not increase and the optical signal to noise ratio (OSNR) of the frequencies generated decrease.

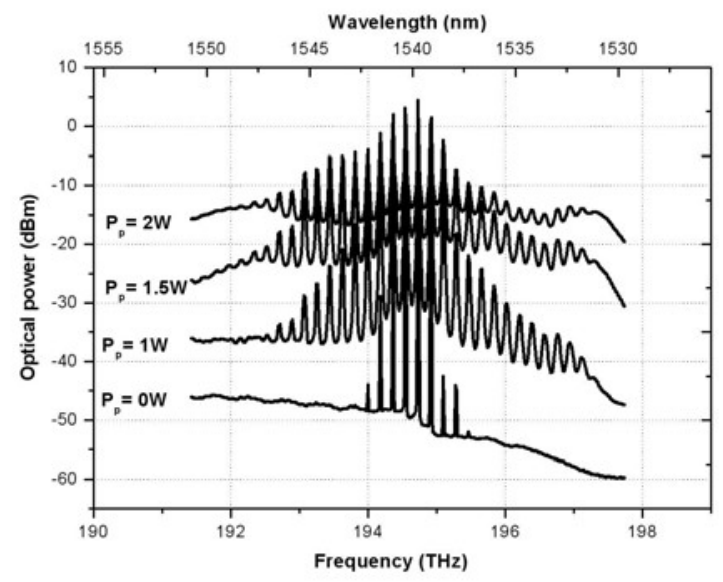

Fig 3.- Output spectra of the laser against different Raman pump power (Raman laser $1455 \mathrm{~nm}$ )

In order to increase the number of frequencies generated we introduced another Raman Fiber Laser (RFL2) pumping at $\lambda \mathrm{p}_{2}=1427 \mathrm{~nm}$ and output power of $\mathrm{Pp}_{2}=1 \mathrm{~W}$ through a 50/50 coupler. Fig 4 shows the new output spectra consisting of 36 equally spaced frequencies covering the frequency range between $192 \mathrm{THz}$ and $198 \mathrm{THz}$ with a spacing of $200 \mathrm{GHz}$.

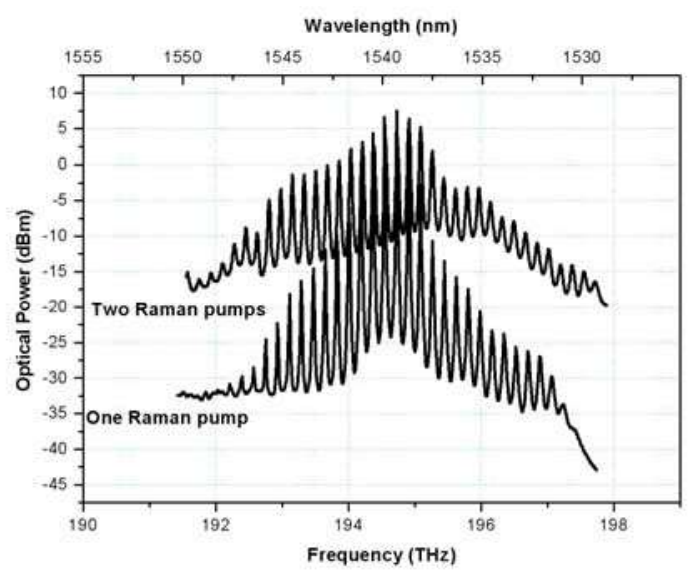

Fig 4.-Comparison of the output spectra of the laser for $1 \mathrm{~W}$ pump power for one $(1455 \mathrm{~nm})$ and two $(1427 \& 1455 \mathrm{~nm})$ pump Raman laser 
With this configuration we obtain a high resolution and tunable multiwavelength fiber source useful for high precision interrogation of fiber Bragg gratings.

Two mechanisms are responsible of the impossibility to increase the efficiency of the system: the strong backward stimulated Brillouin scattering signal generated in the fiber due to the narrow linewidth of the used lasers $(150 \mathrm{kHz})$ that reduces the FWM generation, and the dispersion properties of the fibers that implies the satisfaction of the phase matching condition only in the zero dispersion wavelength region [11].

\section{CONCLUSIONS}

In summary, we have experimentally demonstrated a multiwavelength fiber source based on cascaded four-wave mixing in two semiconductor optical amplifiers (SOAs) enhanced by Raman in a dispersion-shifted fiber. The multiwavelength fiber source generated consists of 36 equally spaced and the spacing can be tuned between 50 and $200 \mathrm{GHz}(0.4 \mathrm{and} 1.6$ $\mathrm{nm})$. The source covers a range from $191.56 \mathrm{THz}(1565 \mathrm{~nm})$ to $197.88 \mathrm{THz}(1515 \mathrm{~nm})$ for a channel spacing of 200 $\mathrm{GHz}$, sweeps with the same spacing over $3 \mathrm{~nm}$ and is continuously monitored in frequency along the sweeps. One step to make the source cheaper could be to replace the Tunable Lasers by simple DFB lasers, and act on their temperature and current. This multiwavelength fiber laser source could be useful for simultaneous and high precision interrogation of a network of FBGs.

\section{Acknowledgments}

We acknowledge financial support from the Ministerio de Educación y Ciencia through the projects TIC2003-01869, TEC200609990-C02-01TCM and the corresponding FPI contract, the support from the Comunidad Autónoma de Madrid through the project FACTOTEM CM S-0505/ESP/0417, and the support from Social European Fund through the grant program I3P of the CSIC.

\section{REFERENCES}

1. A. Bellemare, M. Karásek, M. Rochette, S. LaRochelle and M. Têtu, "Room Temperature Multifrequency ErbiumDoped Fiber Lasers Anchored on the ITU Frequency Grid," J. Ligthwave Technol., 18(6), 825-831 (2000)

2. X. Dong, P.Shum, N.Q.Ngo, and C.C.Chan, "Multiwavelength Raman fiber laser with a continuously-tunable spacing," Opt. Express, 14(8), 3288-3293 (2006)

3. X. Feng, H. Tam, W. Cheng, P.K.A. Wai, "Multiwavelength fiber lasers based on multimode fiber Bragg using offset launch technique," Opt. Comm., 263, 295-299 (2006).

4. X. Yang, X. Dong, S. Zhang, F. Lu, X. Zhou and C. Lu, "Multiwavelength erbium-doped fiber laser with $0.8 \mathrm{~nm}$ spacing using sample Bragg grating and photonic crystal fiber", IEEE Photon. Technol. Lett. 17, 2538-2540 (2005)

5. I. Evans and M. Connelly, "Multiwavelength Mode-locked Fiber Ring Laser with a Lyot filter and a Hybrid Gain Medium", OSA WD6

6. C.S. Kim, R.M. Sona, J.U. Kang and J.B. Khurgin, "Novel multiwavelength cascade-Raman source based on tunable fiber Sagnac loop filter," Tech. Dig. OFC 2002, paper WJ1 (2002)

7. S. Yamashita and K. Hotate, "Multiwavelength erbium-doped fiber laser using intracavity etalon and cooled by liquid Nitrogen," Electron. Lett. 32, 1298-1299 (1996)

8. E. Rivera and D.J. Thomson, "Accurate strain measurements with fiber Bragg sensors and wavelength references," Smart Mater. Struct., 15, 325-330 (2006)

9. Standard reference material 2517a, "High Resolution Wavelength Calibration Reference for 1510nm-1540nm Acetylene ${ }^{12} \mathrm{C}_{2} \mathrm{H}_{2}$," National Institute of Standard and Technology (2000)

10. S. Martín López et al., "Reference frequency generation using Raman enhance four-photon mixing," Appl. Opt. 43, 3185-3190 (2004)

11. K. Inoue, "Four-Wave Mixing in an Optical Fiber in the Zero-Dispersion Wavelength region". J. Lightwave Technol., vol.10, pp. 1553-1561. (1992) 